\title{
First-order agent-based models of emergent behaviour of Dictyostelium discoideum and their inspiration for swarm robotics
}

\section{A selection of aggregation phase behaviour with biological illustrations}

\author{
Mohammad Parhizkar $^{1}$. Giovanna Di Marzo Serugendo ${ }^{1} \cdot$ Jahn Nitschke $^{2} \cdot$ Louis Hellequin $^{2} \cdot$ Assane Wade $^{1}$. \\ Thierry Soldati ${ }^{2}$
}

Received: 7 June 2020 / Accepted: 13 September 2020 / Published online: 23 October 2020

(c) The Author(s) 2020

\begin{abstract}
Collective behaviour in nature provides a source of inspiration to engineer artificial collective adaptive systems, due to their mechanisms favouring adaptation to environmental changes and enabling complex emergent behaviour to arise from a relatively simple behaviour of individual entities. As part of our ongoing research, we study the social amoeba Dictyostelium discoideum to derive agent-based models and mechanisms that we can then exploit in artificial systems, in particular in swarm robotics. In this paper, we present a selection of agent-based models of the aggregation phase of $D$. discoideum, their corresponding biological illustrations and how we used them as an inspiration for transposing this behaviour into swarms of Kilobots. We focus on the stream-breaking phenomenon occurring during the aggregation phase of the life cycle of $D$. discoideum. Results show that the breakup of aggregation streams depends on cell density, motility, motive force and the concentration of cAMP and CF. The breakup also comes with the appearance of late centres. Our computational results show similar behaviour to our biological experiments, using Ax2(ka) strain. For swarm robotics experiments, we focus on signalling and aggregation towards a centre.
\end{abstract}

Keywords Multi-agent models $\cdot$ Self-organisation $\cdot$ D. discoideum $\cdot$ Quorum sensing $\cdot$ Swarm robotics $\cdot$ Kilobots

\section{Introduction}

The work reported in this paper is part of a larger research project, in which we aim at deriving engineering principles inspired by Dictyostelium discoideum to develop collective

This research is supported by the Swiss National Science Foundation (SNSF) [205321_179023].

This work was presented in part at the 3rd International Symposium on Swarm Behavior and Bio-Inspired Robotics (Okinawa, Japan, November 20-22, 2019).

Giovanna Di Marzo Serugendo

Giovanna.Dimarzo@unige.ch

Mohammad Parhizkar

Mohammad.Parhizkar@unige.ch

Jahn Nitschke

Jahn.Nitschke@unige.ch

Louis Hellequin

Louis.Hellequin@unige.ch adaptive artificial systems, (e.g. swarm robotics) [27, 30]. During the transition from growth to development, the ability of $D$. discoideum cells to switch from a unicellular mode of life to a multicellular entity makes it an ideal organism to analyse social and self-organised behaviours and different levels of emergent properties: first- and second-order. First-order level of emergent behaviour refers to swarmlike behaviour, where the emergent properties arise from the collective behaviour of individual entities. We observe this behaviour during the aggregation phase (e.g. streaming)
Assane Wade
Assane.Wade@unige.ch
Thierry Soldati
Thierry.Soldati@unige.ch
1 Centre Universitaire d'Informatique, University of Geneva, Geneva, Switzerland
2 University of Geneva, Geneva, Switzerland 
of $D$. discoideum lifecycle. Second-order level of emergent behaviour refers to individual entities, organising themselves into "super-organisms", themselves displaying a swarm-like behaviour. We observe this behaviour during the migration phase of $D$. discoideum lifecycle, where slugs behave like swarms, while each slug is composed of cells each retaining their individual behaviour, which produces the swarm-like behaviour of the slugs. The key behaviours are cell proliferation, cell-cell adhesion, signalling via extracellular factors, cell death, and cell differentiation as well as differential cell movement, or stream-breaking.

In previous works, we analysed $D$. discoideum life cycle, and provided two agent-based models: (1) streams formation and centres establishment based on six chemical signals [28] and stream-breaking [31] during the aggregation phase; (2) a second model focusing on the slug migration phase of $D$. discoideum, highlighting slugs locomotion, phototaxis and slugs' merging [29].

This paper provides a selection of models, their biological illustrations and swarm robotics experiments for the aggregation phase (first-order emergent behaviour), namely: stream-breaking phenomenon, and swarm robotics aggregation towards the centre. The complete sets of models, results, biological validation and swarm robotics experiments can be found in [26].

We first briefly review the life cycle of $D$. discoideum in Sect. 2, and summarise related works in Sect. 3. We then focus on our new model of the aggregation phase, tackling stream-breaking occurrence in relation with cell motility and resistance and the appearance of late centres in Sect. 4. Simulations, results and biological illustrations can be found in Sects. 5 and 6, respectively. We then show how to transpose some of this behaviour into swarm robotics in Sects. 7 and 8. Finally, Sect. 9 concludes the paper.

\section{D. discoideum life cycle}

Dictyostelium discoideum is a eukaryote, a social amoeba, that feeds on bacteria in the top few centimetres of soil and multiplies by binary fission. The remarkable feature of these cells is that food exhaustion triggers a relatively simple program of multicellular development, to which the starved cells switch their behaviour to in order to survive. This multicellularity in many ways resembles animal development [5]. This ability consists of self-aggregation, dynamic self-assembly, and self-disassembly. Figure 1 illustrates the different stages of the $D$. discoideum life cycle. In the vegetative phase, individual amoeba move around on their own grazing food.

First-order emergent behaviour Once the food is scarce, cells gather in response to cAMP signals, which emanates from the centres. Centres are pacemaker cells which begin

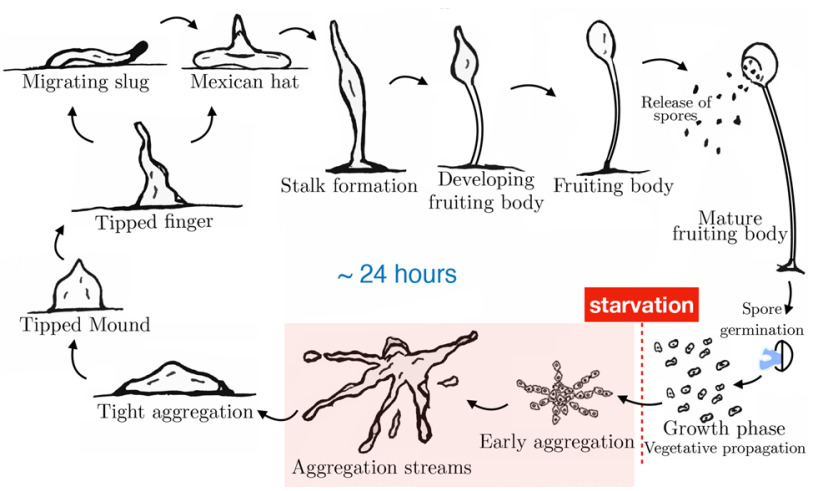

Fig. 1 Schematic diagram of $D$. discoideum life cycle

spontaneously to secrete cAMP. Once $D$. discoideum cells receive the cAMP signal, they start to relay it and aggregate chemotactically towards the higher cAMP concentration area, which has a locally higher cell density [10].

Cells migrate forming patterns, very similar to veins or branches; this phenomenon is called streaming (aggregation phase).

Transition phase Afterwards, streams aggregate and form a hemispherical mass, which is called mound. During this transition phase, cells start to differentiate into several different cell types, e.g., prestalk cells which will make the stalk and the basal disk, upper and lower part of the fruiting body; and prespore cells, which will transform into spores.

Second-order emergent behaviour After aggregation and mound formation, the cells make a coherent and cohesive organisation, which is enclosed by the slime sheath. This superficial morphology is similar to a slug without organs. The multicellular organisation displays phototaxis and thermotaxis behaviour. These two sophisticated slug characteristics lead the cell mass upward towards the surface of the soil in a coordinated way [3, 4]. Additionally, at the migrating stage of $D$. discoideum life cycle, slugs which consist of up to $1 E+$ 5 cells [6] show unique movements without any muscles or nerves. Usually, after about $24 \mathrm{~h}$, the cell mass transforms into a new organisation called a fruiting body consisting of a globe of spores on top of a slender cellular stalk. It holds the spore mass off the ground, for optimal spore dispersal [4]. Eventually, this complex multicellular organisation disperses again, spores germinate, each releasing a new amoeba. At this point, the cells resume their individual behaviour.

\section{Related works}

\subsection{Aggregation phase}

In the field of cell biology, researchers have tried to explain the phenomenon of vein-like streams in $D$. discoideum 
aggregation phase. For instance, Kriebel et al. works [16, 17] illustrate the results of a set of experiments, which study the relationship between the localisation of cAMP and stream formation. They also demonstrate the polarisation and directed migration of cells as a result of asymmetrical distribution of adenylyl cyclase (ACA) at the back of cells.

Several models describe the aggregation phase of $D$. discoideum with formation of cAMP waves [20, 22, 24]. Each model has a distinct explanation for the homogeneity and the generation of aggregation waves. Mackay [21], Vasieva et al. [41], and Van Oss et al. [40] describe discrete models, where each cell has three status: sensitive, refractory and rest. "Local excitation and global inhibition" is used to model the intracellular gradient of chemical secondary messengers that result from the sensing by $D$. discoideum of an extracellular gradient of chemoattractant [18, 19, 25]. This translation of an external chemical cue into an internal gradient leads to cell polarisation in the direction of motion.

Compared to these models, we provide here a novel, integrated model that starts from pre-aggregation and goes up to streaming and stream-breaking processes using the cells' ability to detect and to respond to cells' local density. The model makes use of the most significant signals released by the cells, PSF, CMF, Adenosine, PDE, CF and cAMP. It brings in a series of quorum sensing algorithms for decentralised collective decisions based on various gradients provided by the above signals.

Dallon et al. [8] mathematically modelled the effects of CF using two different models: a cellular automaton model and a discrete continuum hybrid model. Kessler and Levine [13] proposed a discrete and straightforward model to produce and sense cAMP for each cell, which is successful in stream-formation. Each amoeba is considered as a simple component and the cell's behaviour is simulated by a kit of simple rules. This model has been used later by Alameida et al. [1]. The main goal of their model is to lead to the emergence of self-organising intricate regular patterns. None of these models uses a combination of signals and their effect on cells density, on the motive force of individual cells, on late centres emerging to evade the establishment of unreasonably large fruiting bodies, and finally on stream-breaking.

\subsection{Swarm robotics}

Yu et al. [43] present a bio-inspired hierarchical approach for distributed multi-agent systems, implementing an implicit leadership algorithm inspired by Couzin et al. [7].

Swarmanoid [9] is a decentralised system exhibiting selforganised behaviour. In this project, researchers go beyond state of the art in swarm robotics by developing a heterogeneous swarm robotic system made of many robots of different types.
Mathews et al. [23] introduce a new concept of robots with mergeable nervous systems (MNS robots). The main goal is to provide components of centralisation in the organisation of a robot swarm without wasting the advantages of self-organisation. Thus, in their work, they present a swarm of self-assembling robots, which operates in two dimensions. Based on their work, when robots physically dock, they share a joint architecture for sensing the environment and for the decision-making process. They call it the robot's nervous systems, where one robot acts as a brain unit to make the decisions.

Other swarm control strategies we can mention are: the trophallaxis inspired strategy [34] and the vector-based approach [37]. Through the 'LaRoSim' multi-agent simulation platform, the authors simulate a scenario of collective cleaning task. The simulated robots are designed accordingly to the capabilities of the real JASMINE [14, 15] and I-SWARM [35] robots.

Valentini et al. [38, 39] propose an iterative strategy to tackle the best-of-n decision problem in a swarm of robots. Each robot examines all available choices, measures the quality of each option. Then, the robot makes an autonomous decision and broadcasts the decision to its neighbors. Finally, the authors implement decision-making strategy using a swarm of 100 Kilobots to evaluate its robustness. The strategy involves the motors, the light sensor, and the infrared system of the Kilobots.

Research in swarm-robotics investigates various organisations, from centralised or hierarchic, to decentralised ones, with homogeneous or heterogeneous robots, aiming at various activities (solving specific tasks or decision problems). We are interested in the full process from identifying firstand second-order emergent behaviour in natural systems and how to translate them into artificial ones, e.g. swarm robotics.

\section{First-order collective behaviour: agent-based model of stream-breaking}

In previous works, we modelled the amoeba cells aggregation using a self-organised model for first-order collective behaviours [28, 29]. The model considers two fundamental and essential factors giving rise to streams of cells. First, regular cells relay the cAMP signal during early aggregation. The second factor is that cells move up the chemical gradient from low cAMP concentration to a higher concentration. This directional locomotion of cells towards a source allows the small initial variations in density to be strengthened.

Figure 2 shows the increasing levels of cAMP threshold regulating respectively the appearance of centres, the relay of cAMP by regular cells and the chemotaxis threshold inducing cells movements and streaming [42]. Chemotaxis 


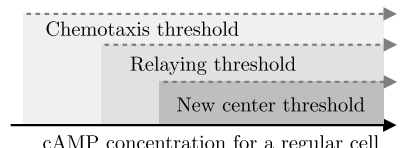

Fig. 2 Different levels of cAMP thresholds

in D. discoideum is the movement of the amoebae towards a higher concentration of a chemical (usually towards the source of a gradient), which in starving $D$. discoideum is cAMP.

\subsection{Streaming and stream-breaking}

Our main intention is to complete our previous firstorder agent-based model [29] and further investigate the stream-breaking process. In particular, we are interested in understanding the process of late centre formation and how, together with the forces involved in the motion of the cells, it is linked with the stream-breaking phenomenon. As illustrated in our previous work [29], the model shows first that the system is capable of producing streaming once cell movement is triggered, and second, it displays waves of cell movement during the $D$. discoideum aggregation phase. This paper shows that the stream-breaking phenomenon is augmented and sustained by the capability of cells to relay the cAMP signal to their local neighbours. It also shows the relationship between cAMP, CF and motive force. During the aggregation phase, when cells aggregate towards a source, the moving cells change their location towards an area, which has a locally higher cell density. Therefore, the density of the cells in those areas increases. Consequently, they attract additional cells. Cells at high-density produce more $\mathrm{CF}$, which in combination with motive force causes stream breakup.

Our model integrates three main elements: (1) modelling of the force involved in the movement of the cells (motive force) and resistance of cells; (2) quorum sensing-identify threshold of concentration of cAMP, below which cells start losing motive force, and threshold of $\mathrm{CF}$, above which the cell-intrinsic resistance increases because of the high density of cells; (3) emergence of late centres further attracting cells.

Quorum sensing In our model, individual cells use quorum sensing to collectively identify cAMP and CF threshold to compute the motive force.

Late centres At high cell density, aggregation streams break into smaller groups towards an aggregation centre [12]. In some cases, these groups then form a separate fruiting body, and in some others, they merge again with a more significant aggregation mass. We call these centres, "late centres", because they start signalling as centres much later in the process.

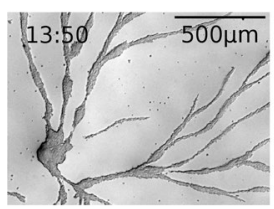

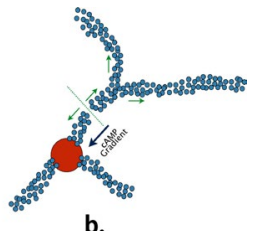

Fig. 3 (a Stream formation and stream breaking of Ax2(ka) cells; b rpresentation of stream-breaking mechanism: one centre (red) and 200 attracted cells (blue)

Motive force $(\mathrm{mf})$ ach cell is capable of moving against its own intrinsic resistance, using the motive force, generated by the collective of cells attracted to cAMP.

We treat the motive force of individual cells as a function of time and of the concentration level of cAMP. Cells that sense the same amount of concentration of cAMP generate the same motive force $\left(P_{1}\right)$. Using the concentration of $\mathrm{CF}$, the local density of cells changes the resistance force $\left(P_{2}\right)$ in the streams (Eq. 1).

$P_{1_{t}}=\left\{\begin{array}{l}\alpha * C_{\mathrm{cAMP}_{t}}, \text { if cAMP } \geq T_{\text {pulse }} \\ m f_{t-1}-\beta * t, \text { if } c A M P<T_{\text {pulse }}\end{array}\right.$

$P_{2_{t}}=\omega * C_{C F_{t}}$, see Table 1 for initial values

Here $C_{\text {cAMP }}$ denotes the value of concentration of cAMP, which varies based on the distance from the centre. $C_{\mathrm{CF}}$ is the value of the concentration of $\mathrm{CF}$, which has different values in different cell densities. The constant coefficients $\alpha$ and $\omega$ are used to calculate the $P_{1}$ and $P_{2}$ respectively, for each cell, and $\beta$ is the constant coefficient for time. When the concentration of cAMP is greater or equal to $T_{\text {pulse }}$, the motive force is linear to the concentration of cAMP. If the concentration of cAMP is less than $T_{\text {pulse }}$, the motive force value decreases in each time step.

Eventually, the streams start to make a gap (see Fig. 3), and it breaks, if in a cell, the motive force is smaller than the resistance force of the cell $P_{1}<P_{2}$.

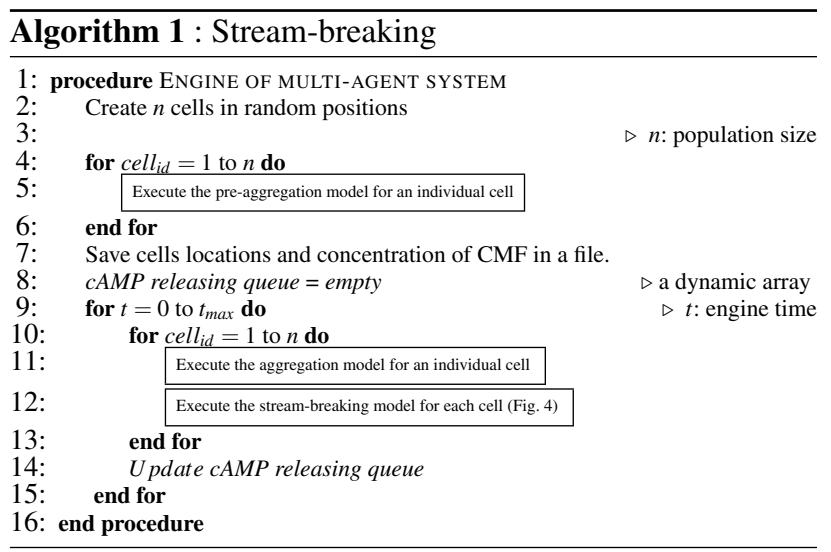




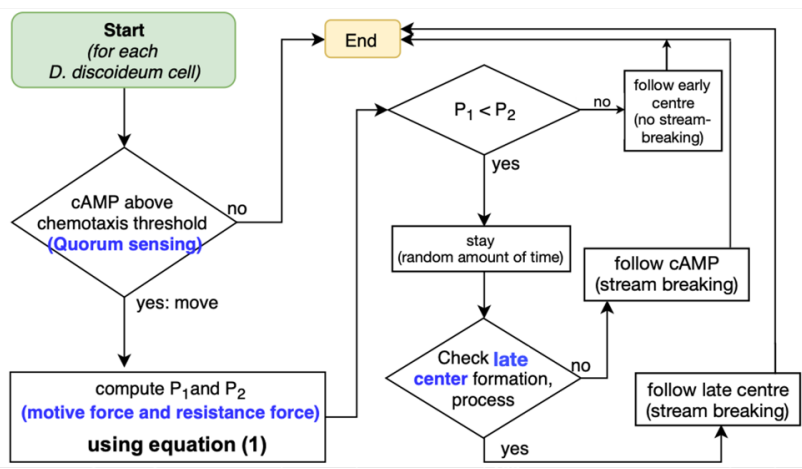

Fig. 4 Stream-breaking model for each individual cell

Algorithm 1 delineates the individual cells behaviour for stream formation. We updated our previous model with a stream-breaking behaviour using the three elements above (quorum sensing, the motive force and late centres), described explicitly in Fig. 4.

\section{First-order collective behaviour: simulations and results}

In our model, each cell measures the local concentration of cAMP and responds by moving towards the source of the spatial gradient, when the concentration of cAMP is above the threshold of $1 E-9 \mathrm{nM}$, as specified in Table 1. We use a diffusion-based model to simulate cAMP and other chemical factors. The concentration of cAMP decreases away from the source and is degraded by PDE as it diffuses away.

The basic algorithm of the program is shown in Fig. 4 and Algorithm 1. The model does include simple chemotaxis towards nearby groups of cells. The two parameters: cell density and the position of early centre (randomly chosen) were modified in each simulation to examine the influence of these changes on the final number of aggregation territories. Five typical examples of our simulation results can be seen in Figs. 5, 6, 7, 9 and 10. In these examples, there were 1000 starved cells in blue and one spontaneous signalling cell in red. The movies are available on-line on the project's website ${ }^{1}$.

\subsection{Stream-breaking: emergence of late centres}

When cell density in a stream becomes too high around an early declared centre, the concentrations of cAMP and CF increase. Using quorum sensing, cells identify when the density threshold is reached, and whether potential late centres

\footnotetext{
${ }_{1}$ https://www.unige.ch/cui/cas/research/dicty
}

are needed. Depending on locally perceived (low) concentrations of Adenosine and CMF, a cell may decide to turn itself into an aggregation (late) centre (see Figs. 5, 6, 7, 8, and 10).

Stable and unstable late centres After stream-breaking, two different kinds of aggregation (late) centres can emerge: the stable centres and the unfixed centres. In most of our simulations, the late centre and the group of cells around it are not stable. The cells and even the late centre are still attracted to the stronger group, and they still move towards the early centre (see Figs. 5, 6). In experiments with more cells, we observed that the late cell mass moves in a slower manner towards the early centre (Fig. 8 with 4000 cells).

In other cases, when there are enough cells around the late centre and it is far enough from the early centre, the late centre is mostly stable. In this case, the cells are attracted more to the late centre see Fig. 7, 10).

\subsection{Stream-breaking: no emergence of late centres}

During stream-breaking phenomena (Fig. 9), cells make a late cluster by gathering somewhere far from the early centre, but no late centre appears. There might be two explanations for this lack of late centres appearance:

1. In aggregation territories, Adenosine is diffusing outwards from the early formed centres, which prevents the formation of late centres.

2. The reason also could be insufficient number of cells in this cluster to cause any of the cells to declare itself a late centre spontaneously.

\subsection{Initial conditions}

Table 1, inspired by Mackay works [21], illustrates the initial values of parameters, which were used and explained in more detail in our previous work [29].

\section{First-order collective behaviour: biological illustration}

To investigate the biological relevance of the simulated behaviours and the resulting emergent properties, a range of biological experiments were carried out in parallel. Dictyostelium discoideum, strain Ax2(ka) [2], was cultured in Petri dishes (Corning, $100 \times 20 \mathrm{~mm}$ ) at $22^{\circ} \mathrm{C}$ in H5Lc medium. The developmental cycle was initiated by exchanging the culture medium with the SorMC (15 mM $\mathrm{KH}_{2} \mathrm{PO}_{4}, 2 \mathrm{mM} \mathrm{Na} \mathrm{HPO}_{4}, 50 \mu \mathrm{M} \mathrm{MgCl}_{2}, 50 \mu M \mathrm{CaCl}_{2}$, nominal pH 6.0) non-nutrient buffer, concomitantly with the plating of 2 million cells on a thin layer of $3 \mathrm{~mm}$ of 

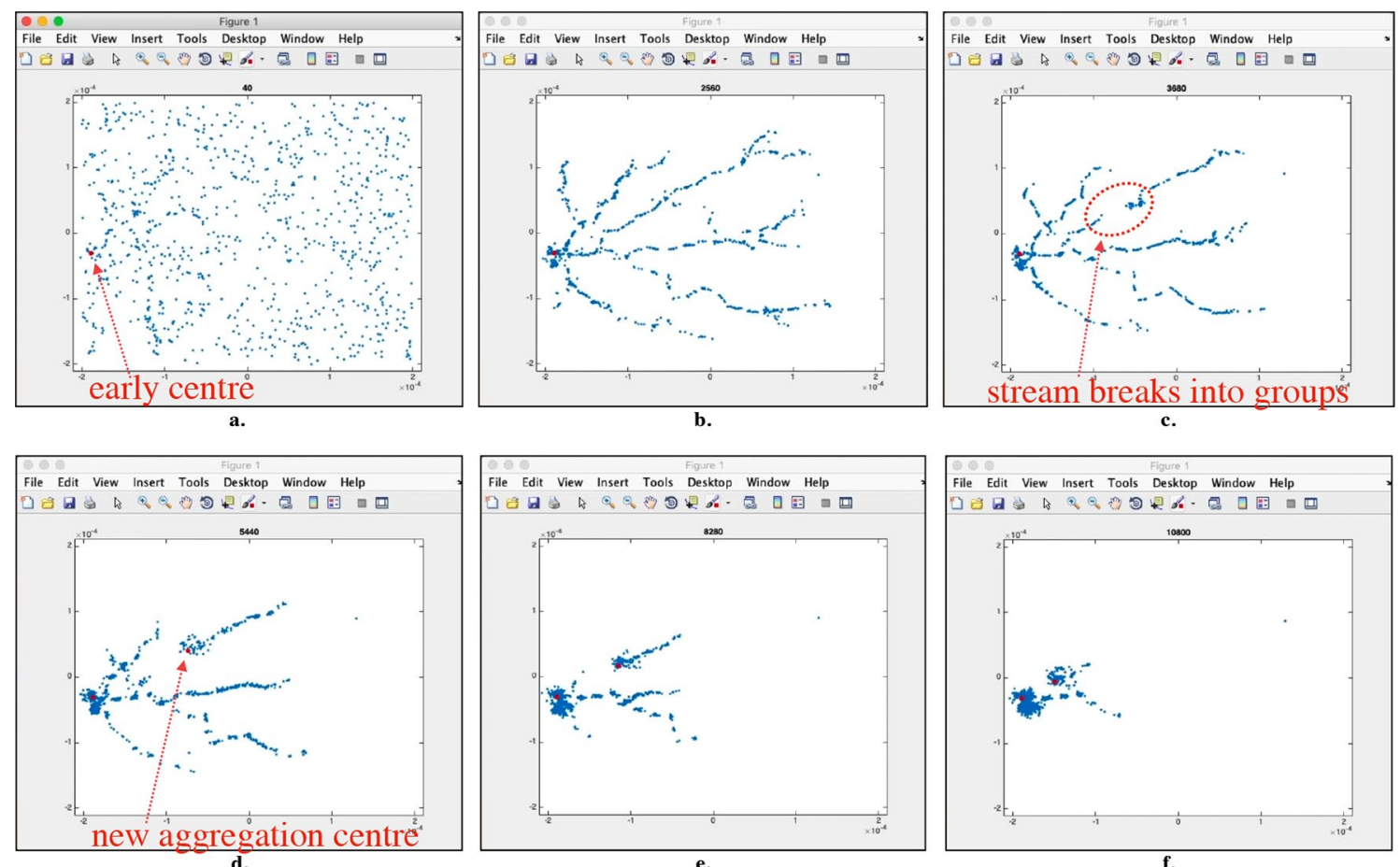

Fig. 5 Stream-breaking: 1000 normal cells, one early spontaneous centre-late centre formation appears in $d$, however, the late centre is not a stable centre
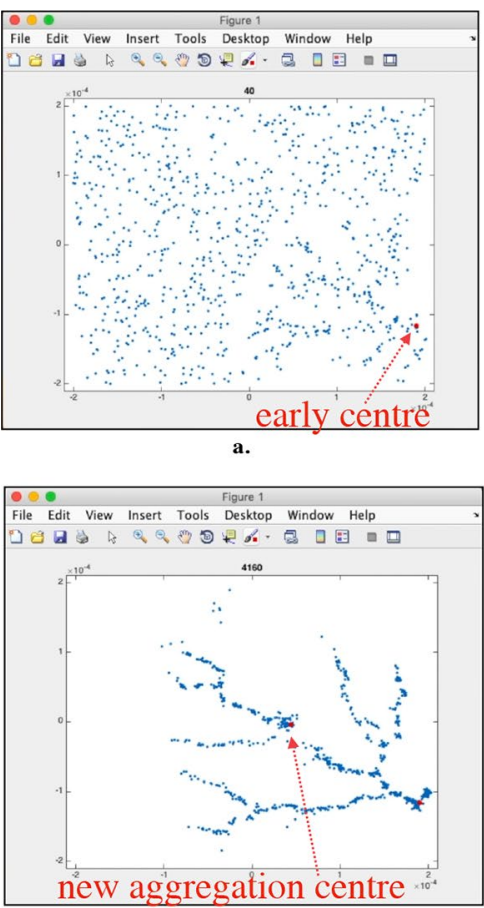

d.

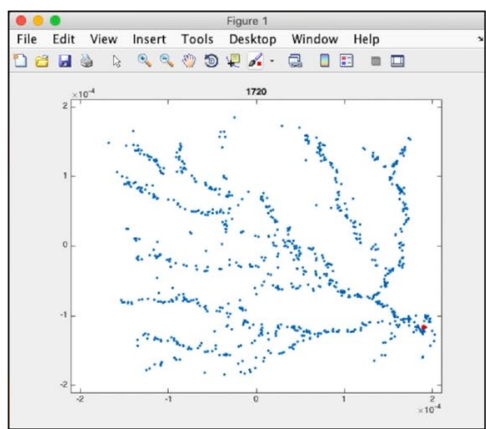

b.

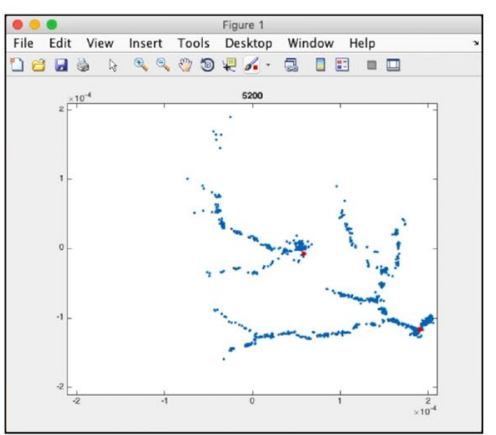

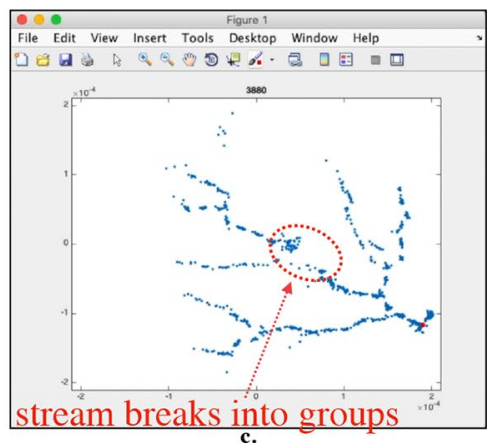

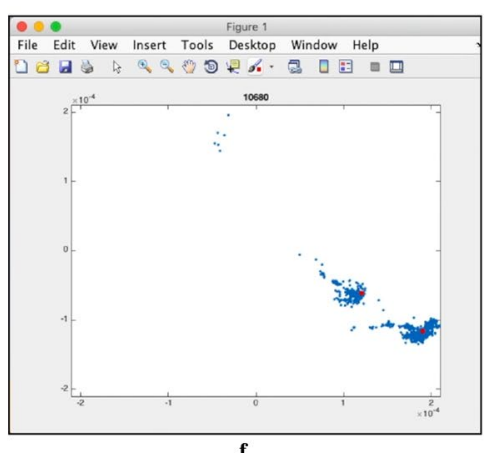

Fig. 6 Stream-breaking: 1000 normal cells, one early spontaneous centre-late centre formation appears in $d$, however, the late centre is not a stable centre 

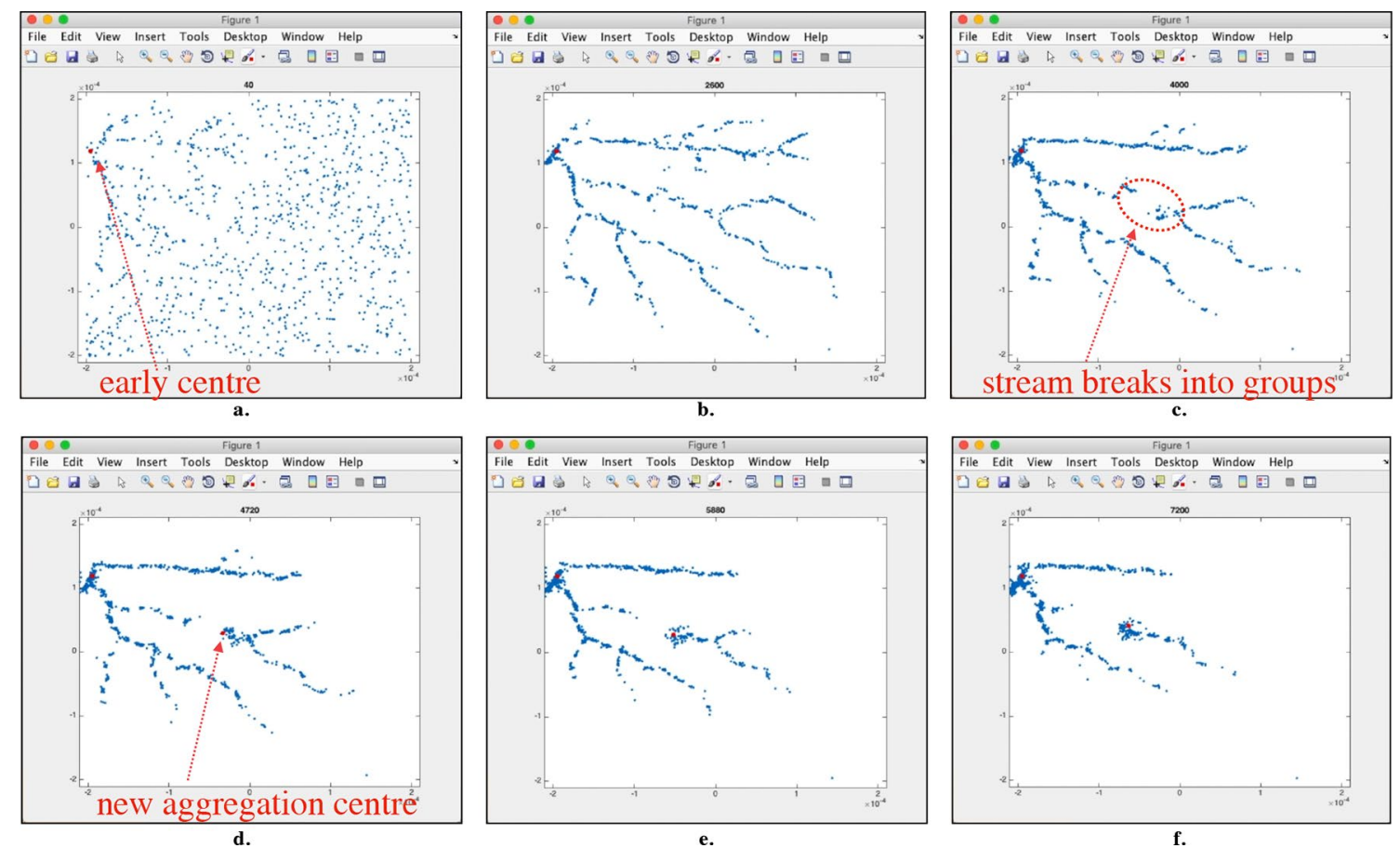

Fig. 7 Stream-breaking: 1000 normal cells, one early spontaneous centre—in $d$, a late stable centre appears
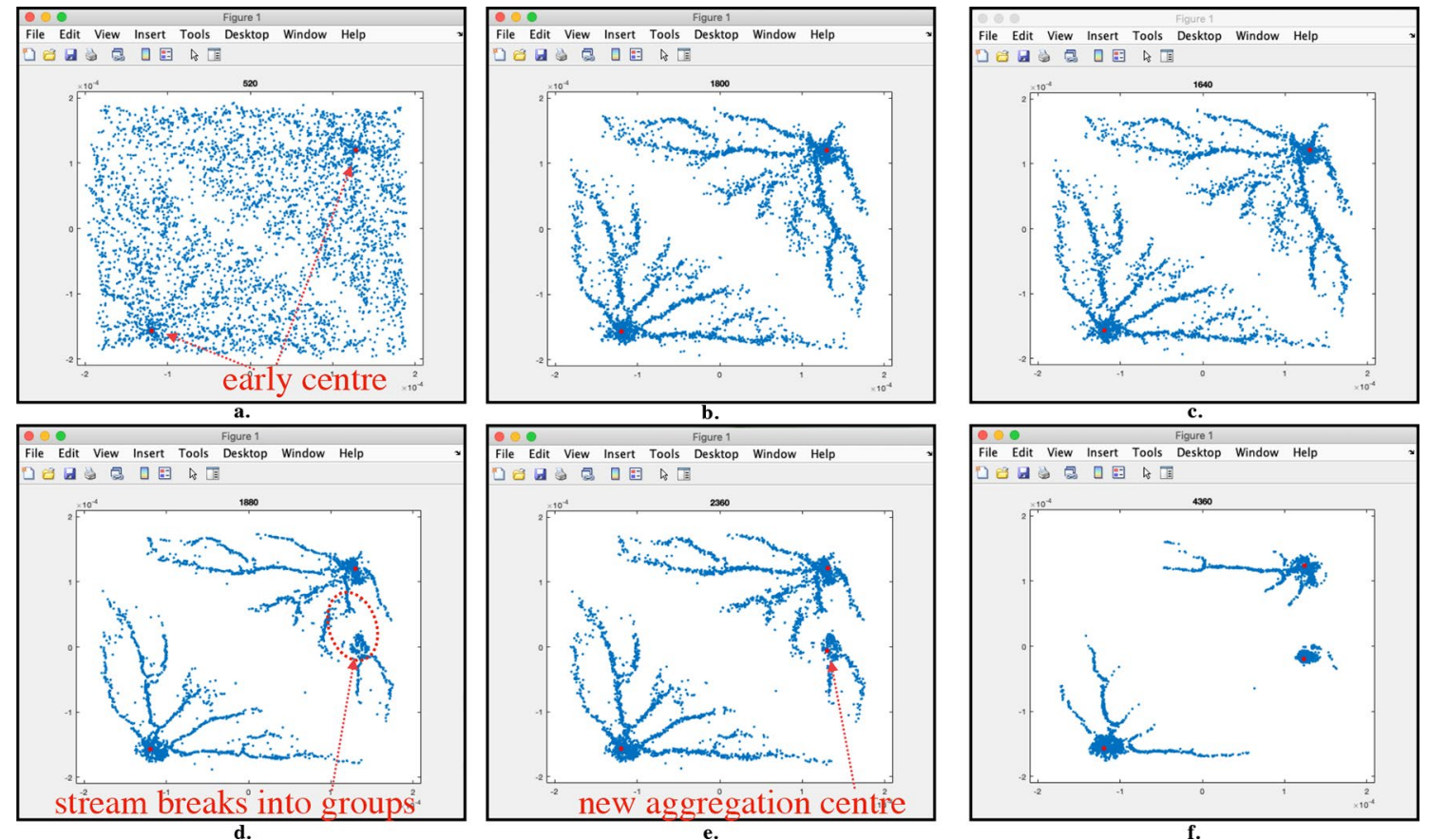

Fig. 8 Stream-breaking: 4000 cells, 2 early centres-in $d$ a late centre appears, it is not stable and moves slowly towards an early centre

BactoAgar (2\% in SorMC) in an ibidi glass-bottom dish (ibiTreat " $\mu$-dish $35 \mu \mathrm{m}$ high", $35 \times 12 \mathrm{~mm}$ ). The cells were imaged using differential interference contrast microscopy with a Leica DMI8 widefield microscope $(10 \times$ objective, tile scans of $10 \times 10$ field of views, one image every $5 \mathrm{~min}$ ) for up to $18 \mathrm{~h}$ (see Fig. 10). 

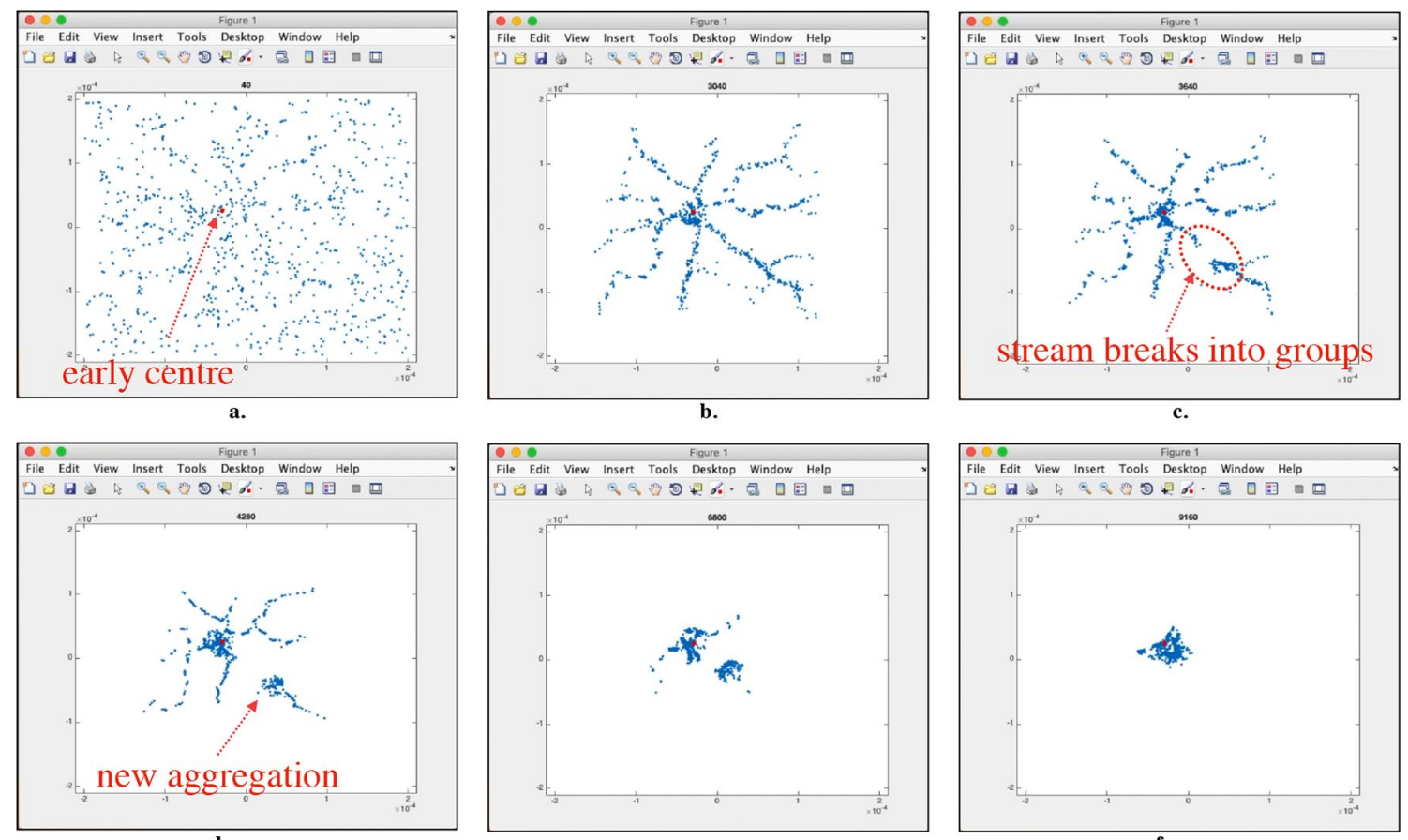

Fig. 9 Stream-breaking: 1000 normal cells, one early spontaneous centre-in this experiment, there is no late centre formation. Thus, the small group is still attracted to the bigger group. The two groups merge together in $f$
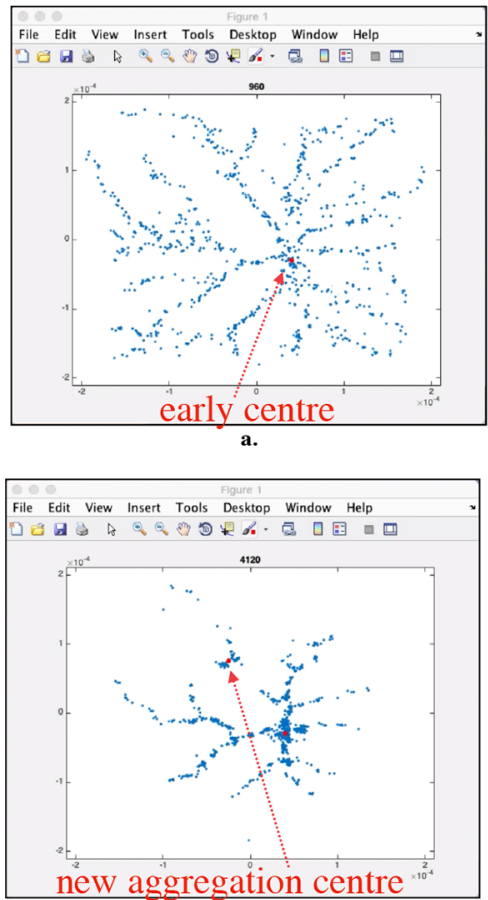

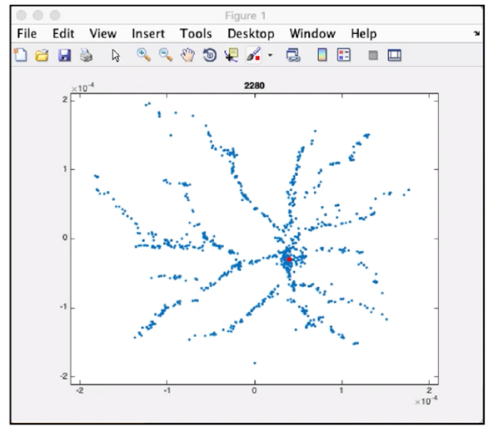

b.

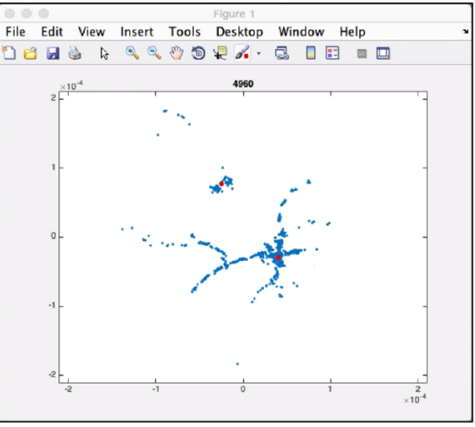

e.

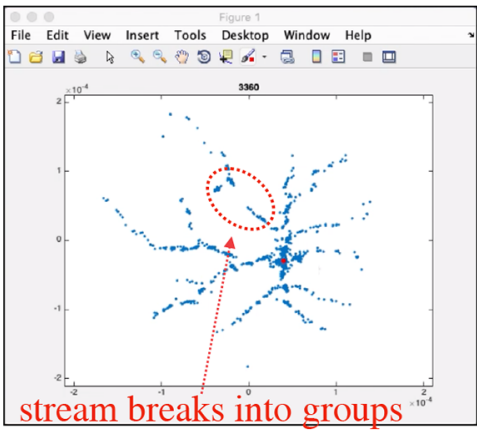

c.

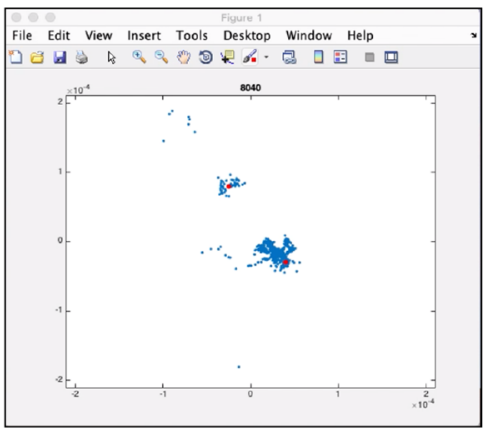

Fig. 10 Stream-breaking: 1000 normal cells, one early centre — late stable centre appears in $d$ 
Table 1 Simulation initial parameters [21]

\begin{tabular}{ll}
\hline Parameter & Initial value \\
\hline No. of molecules release by each cell & $1 E+7$ \\
Diffusion constant of cAMP & $9.7 \times 1 E-6 \mathrm{~cm}^{2} / \mathrm{s}$ \\
$\tau_{0}$ & $1 E-10 \mathrm{~s}$ \\
$T_{\text {Relay }}, T_{\text {Chemotaxis }}$ & $8 \times 1 E-9 M, 1 E-9 M$ \\
$T_{\text {Pulse }}$ & $9 \times 1 E-9 M$ \\
Delay for relay & $15 \mathrm{~s}$ \\
Refractory period & $10-3$ min \\
Refractory period for chemotaxis & $100 \mathrm{~s}$ \\
Chemotaxis step & $20 \mu \mathrm{m}$ in $100 \mathrm{~s}$ \\
Random motion speed & $5 \mu \mathrm{m} / \mathrm{min}$ \\
Area & $4 E-3 \times 4 E-3$ \\
Late centre formation, & $2 \times 1-6$ \\
cAMP threshold & $1 E-9 \mathrm{M}$ \\
Adenosine threshold & $1 E-5 \mathrm{M}$ \\
CF threshold, CMF threshold & $1 E-5 \mathrm{M}, 1 E-5 \mathrm{M}$ \\
Released adenosine molecule & $3 \times 1 E+3$ \\
Max time simulation, $t$ & $3 \times 60 \times 60,6 \times 60$ \\
Each time step & Equals to $1 \mathrm{~min}$ \\
$\alpha, \beta, \omega$ & $1 E-2,1,9 \times 1 E-3$ \\
Michaelis-Menten constant for PDE & $K_{\mathrm{m}}$ \\
Maximum activity of PD & $V_{\max }$ \\
\hline
\end{tabular}

We observed the complete developmental cycle starting with the unicellular amoebae and covering streaming, mound formation, slug motility and culmination in a fruiting body. Stream-breaking occurred both along linear streams and preferentially where streams join, resulting in the formation of similarly sized and spaced late centres and mounds (Fig. 11). Similar streaming and stream-breaking behaviour were also visible in our simulations.

Late centres resulting from stream-breaking often remained stable, formed independent mounds, and then progressed through the final stages of the developmental cycle (Fig. 11). Analogously, stable secondary centres were also observed in simulations (Figs. 7, 10). In some circumstances, the late centres gave rise to unstable mounds which dispersed again into streams and subsequently merged with other attracting stable mounds (Fig. 12). Analogously, such unstable secondary centres that merge with a primary centre, were also observed in simulations, (see Figs. 5, 6, and 8).

\section{Swarm robotics}

We illustrate the transposition of the various models to an artificial system made a Kilobots and results we derived from $D$. discoideum, namely: the robot-robot interaction, synchronisation, leader selection, aggregation toward a selected centre.

Kilobots [32, 33], with a diameter of $3 \mathrm{~cm}$, were developed at Harvard University's Self-organising Systems Research Lab. The main goal was to serve the need for actual experimentation and confirmation of collective algorithms in self-organising systems.

To program Kilobots, we use an overhead infrared transmitter instead of plug-in cable, which allows us to program all the Kilobots collectively once in a time. Kilobots use a pair of vibration motors for stick-slip motion, a reflective

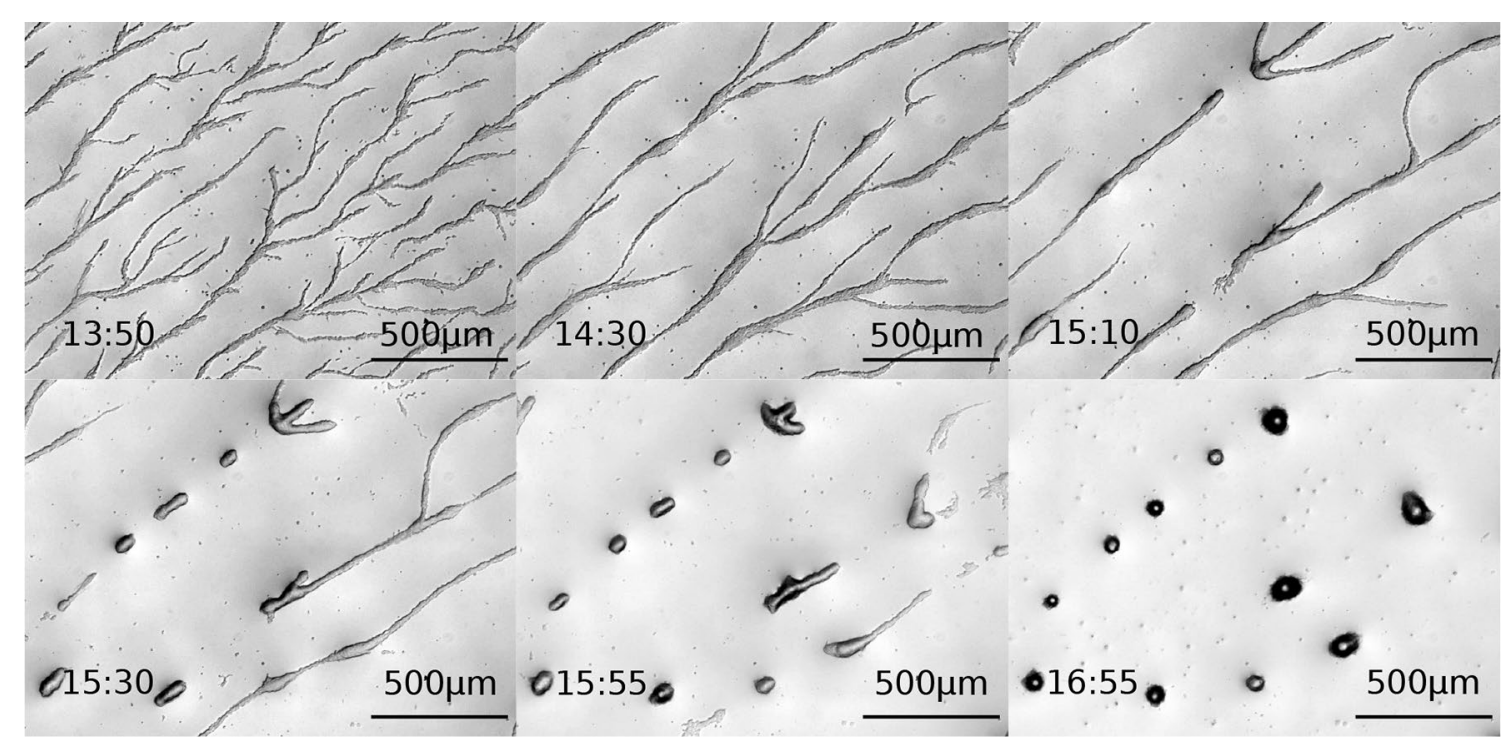

Fig. 11 Streaming and stream-breaking. Cells polarise in streams (13:50-14:30), subsequent stream-breaking and formation of similarly spaced and sized mounds along the stream and stream junctions
(15:10-16:55). Time after onset of starvation is indicated at the bottom left [hh:mm]. Scalebar: $500 \mu \mathrm{m}$ 


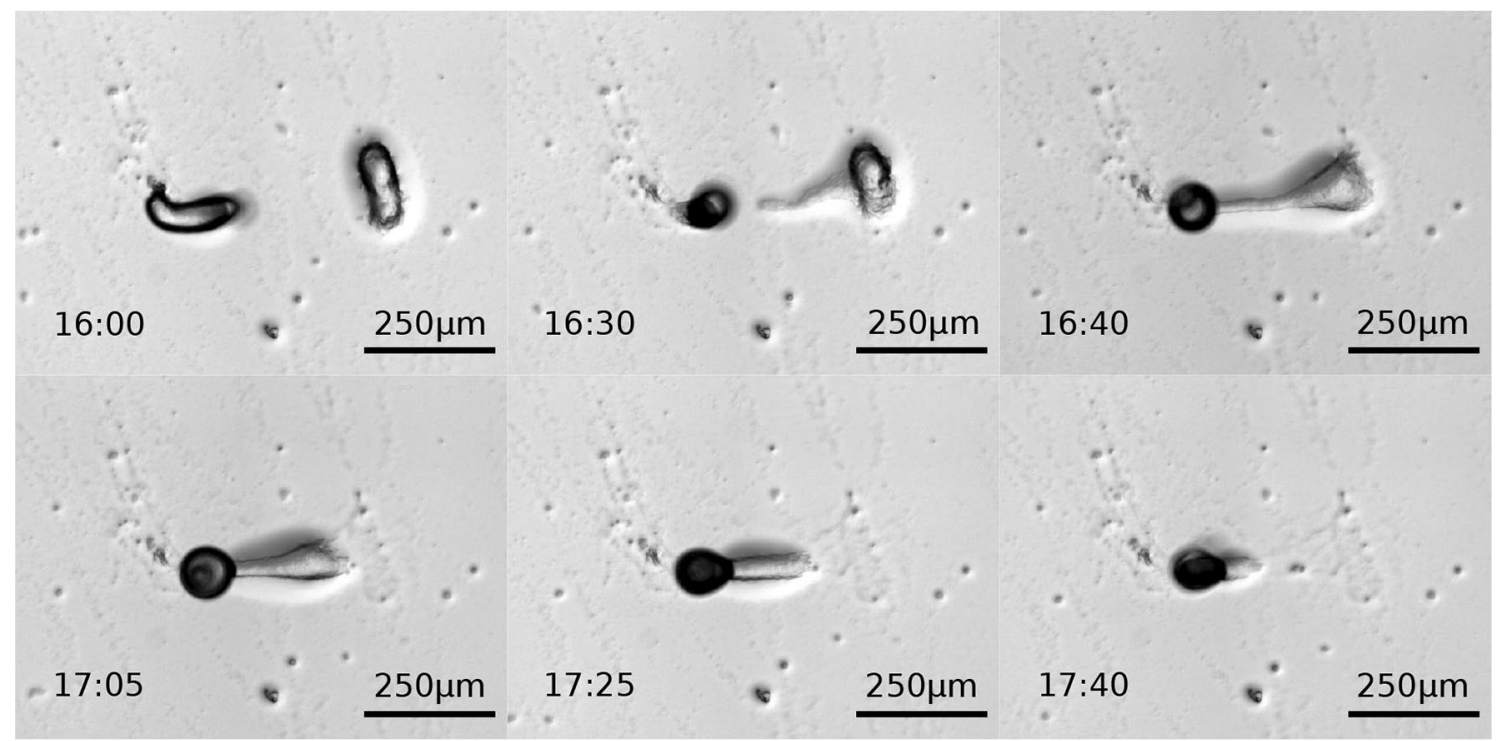

Fig. 12 Centre absorption. The left mound proceeded to the slug stage (16:00), cells from the right mound were attracted by the approaching slug (16:30). The complete cell mass from the right mound streamed towards the left mound (16:40-17:05) and merged

infrared LED, and distance sensing to communicate with other robots in their neighborhoods. Communications are transmitted by pulsing messages in a range of $10 \mathrm{~cm} \mathrm{[32]}$ by infrared LED, which enables the robot to get information uniformly from all directions. All the neighbors in the range from any direction can receive the emitted light, which has been reflected by the surface. Sensing measured distances between neighbors as location feedback helps the robot movement to be relatively correct.

A Kilobot can determine the distance to the message sender based on the signal strength but not the direction change. The infrared receiver assists robots to continuously collect and observe the signal strength to determine the neighbor positions [11].

During this project, all the programs for Kilobots are written in the $\mathrm{C}$ programming language. To transfer the code to a group of self-organised Kilobots, an overhead infrared controller is used, which is connected to the computer by a USB port. After sending the code to the robot, they download the program. Then, the program begins, and the same autonomous act initiates with no other communication from the computer. Kilobots computing capabilities are limited, experiments focus on limited parts of the behaviour, and are studied one at a time.

Functions and complexity Different single functions are used to control the movement and RGB LED, for example, communication with neighbors, movement direction (right, left, straight), blinking after a specific time. The complexity of the program depends on the number of sent messages from the robots to each other.
$(17: 25-17: 40)$. Note the similar volume of both cell masses at the beginning. Time after onset of starvation is indicated at the bottom left of each panel [hh:mm]. Scalebar: $250 \mu \mathrm{m}$

Communication In all experiments, messages are sent every $0.5 \mathrm{~s}$. The message contains 9-bytes, plus a 1-byte for message type and 2-bytes for the checksum.

Synchronisation A Kilobot status has time in $30 \mathrm{~ms}$ since it was last validated. When the Kilobots move, they reset their status after two seconds.

Library There is a small library of $\mathrm{C}$ programming language that is available for Kilobots. It includes some useful functions for LED colours, establishing motors, and bearing and broadcasting messages.

\section{Transposing models to Kilobots: first-order emergent behaviour}

We discuss a selection of behaviours transposed into Kilobots. Videos of these experiments are freely available ${ }^{2}$.

\subsection{Signalling and synchronisation-leader selection}

In the field of swarm robotics and sensor networks, synchronisation is essential to coordinate contemporaneous operations with numerous computational agents or robots. In general, each Kilobot maintains a periodic clock. The clock possesses a threshold, which resets the clock when it reaches its maximum. Figure 13 illustrates the synchronisation experiment, which we used with a full pack of 10

\footnotetext{
${ }^{2}$ https://www.unige.ch/cui/cas/research/dicty
} 


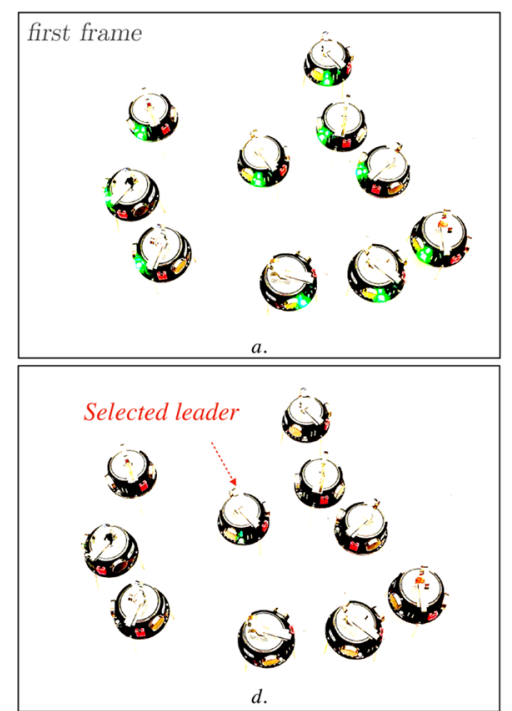

Fig. 13 Kilobots synchronisation and leader selection process: In this experiment, Kilobots synchronise themselves in a swarm. a Each robot generates a random $I D$ and blinks when it's done (RGB LED on-Green). b Each robot propagates its $I D$. The idea is to find the robot with the highest random ID (RGB LED on-Blue). c After the robots shared their $I D$, they elect the robot with the highest $I D$. The leader's LED is off, and the others have green LED. $\mathbf{d}$ Then, the chosen robot (RGB LED on-Green) propagates a message to the whole group (RGB LED-off). The message is to start a random walk after $3 \mathrm{~s}$. e The others receive the message and confirm it by blinking the green light. f Then, the robots change the LED colour to red and begin the movement in a random direction
Fig. 14 Progressive aggregation of Kilobots towards a Kilobot at the centre of the field

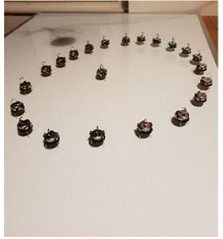

(a)

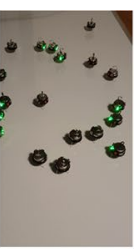

(b)

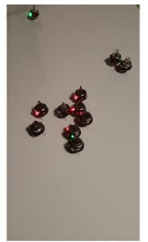

(c)

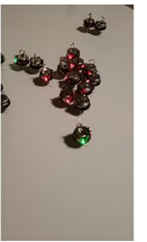

(d)

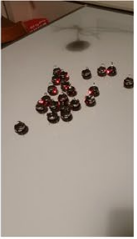

(e)
Kilobots. They communicate with each other, and everyone takes a random ID number. Then, the robot with the highest ID number becomes the leader of the group (with a red LED light), and propagates its role to the whole swarm. Afterward, the robots acknowledge the leader's message, by blinking the green LED light. Next, the leader robot sends another signal, which is a message to begin the random movement after a specific period (using the robot's internal clock), in a synchronised way.

\subsection{Aggregation toward a centre}

Figure 14, from (a) to (e), illustrates the aggregation behaviour with a series of consecutive images. Aggregation is a prevalent biologically inspired task in collective robotics. The task generally involves robots waiting in their pause state, until they receive a signal from a centre. One immobile Kilobot with a specialised program is placed in the middle of the field with the other robots all around (a). This marker continuously broadcasts a message that tells any robots within the communication area that the robot is the centre of aggregation. This message also serves the other robots to identify their distance wrt to that centre. Next, the other robots approach the centre slowly (b)-(d), by just progressing forward, until they have a sufficient satisfactory distance from the fixed Kilobot. At this point they turn their light from green to red (e).

\section{Conclusion}

In studying the aggregation and slug formation phases of D. discoideum, the motive force, which is generated from starved cells is one of the primary elements to be considered [8, 36]. In this study, we explain our integrated model of cells motive force, late centres formation, and stream-breaking, by modelling and simulating key chemical signals propagation: 3', 5'-cyclic adenosine monophosphate (cAMP), Counting Factor (CF), Phosphodiesterase 
(PDE), Prestarvation Factor (PSF), Conditioned Medium Factor $(C M F)$, Adenosine. We enriched our previous models [29] for stream formation during $D$. discoideum aggregation phase with stream-breaking features. We modelled three main elements to produce realistic behaviour: cells motive force, late centres, and quorum sensing. The new version of the model is successful in generating stream branching and stream-breaking. Our complete model illustrates the importance of various parameters such as population size, cell's random motion, cAMP, PDE, $\mathrm{CF}$ activities, and motive force of individual cells, to explain the dynamics of the system.

We complement our simulation results with corresponding biological illustrations. We also show how some parts of the models translate into swarm robotics. We continue implementing our models into Kilobots to investigate how the whole model translates into swarm robotics, in particular with a larger number of Kilobots, and how we can reproduce the transition phase from first- to second-order.

\section{Funding Open access funding provided by University of Geneva}

Open Access This article is licensed under a Creative Commons Attribution 4.0 International License, which permits use, sharing, adaptation, distribution and reproduction in any medium or format, as long as you give appropriate credit to the original author(s) and the source, provide a link to the Creative Commons licence, and indicate if changes were made. The images or other third party material in this article are included in the article's Creative Commons licence, unless indicated otherwise in a credit line to the material. If material is not included in the article's Creative Commons licence and your intended use is not permitted by statutory regulation or exceeds the permitted use, you will need to obtain permission directly from the copyright holder. To view a copy of this licence, visit http://creativecommons.org/licenses/by/4.0/.

\section{References}

1. Almeida S, Dilao R (2015) Modelling Dictyostelium discoideum aggregation through a discrete excitability model with directional sensing. In: Advances in systems and synthetic biology, pp 125-137

2. Bloomfield G, Tanaka Y, Skelton J, Ivens A, Kay RR (2008) Widespread duplications in the genomes of laboratory stocks of Dictyostelium discoideum. Genome Biol 9(4):R75

3. Bonner JT, Lamont DS (2005) Behavior of cellular slime molds in the soil. Mycologia 97:178

4. Brock DA, Gomer RH (1999) A cell-counting factor regulating structure size in Dictyostelium. Genes Dev 13(15):1960-1969

5. Coates JC, Harwood AJ (2001) Cell-cell adhesion and signal transduction during Dictyostelium development. J Cell Sci 114(24):4349-4358

6. Cooper GM (2000) An Overview of Cells and Cell Research. In: The Cell: A Molecular Approach, Chapter 1, 2nd edition. Sunderland (MA), Sinauer Associates. https://www.ncbi.nlm. nih.gov/books/NBK9839/

7. Couzin ID, Krause J, Franks NR, Levin SA (2005) Effective leadership and decision-making in animal groups on the move. Nature 433(7025):513
8. Dallon J, Jang W, Gomer RH (2006) Mathematically modelling the effects of counting factor in Dictyostelium discoideum. Math Med Biol 23(1):45-62

9. Dorigo M, Floreano D, Gambardella LM, Mondada F, Nolfi S, Baaboura T, Birattari M, Bonani M, Brambilla M, Brutschy A (2013) Others: Swarmanoid: a novel concept for the study of heterogeneous robotic swarms. IEEE Robot Autom Mag 20(4):60-71

10. Dormann D, Vasiev B, Weijer CJ (1998) Propagating waves control Dictyostelium discoideum morphogenesis. Biophys Chem 72:21-35

11. Griffith J, Holland J, O'Riordan C (2016) An evolutionary approach to formation control with mobile robots. In: Proceedings of the 8th International Joint Conference on computational intelligence, Vol 1: ECTA. SciTePress Digital Library

12. Jang W, Gomer RH (2008) Combining experiments and modelling to understand size regulation in Dictyostelium discoideum. J R Soc Interface 5(suppl-1):S49-S58

13. Kessler DA, Levine H (1993) Pattern formation in Dictyostelium via the dynamics of cooperative biological entities. Phys Rev 48:4801

14. Kornienko S, Kornienko O, Levi P (2005) Collective AI: context awareness via communication. IJCAI 5:1464-1470

15. Kornienko S, Kornienko O, Levi P (2005) Minimalistic approach towards communication and perception in microrobotic swarms. In: 2005 IEEE/RSJ Int. Conf. Intell. Robot. Syst. IROS. https:// doi.org/10.1109/IROS.2005.1545594

16. Kriebel PW, Barr VA, Parent CA (2003) Adenylyl cyclase localization regulates streaming during chemotaxis. Cell 112(4):549-560

17. Kriebel PW, Barr VA, Rericha EC, Zhang G, Parent CA (2008) Collective cell migration requires vesicular trafficking for chemoattractant delivery at the trailing edge. J Cell Biol 183(5):949-961

18. Krishnan J, Iglesias PA (2003) Analysis of the signal transduction properties of a module of spatial sensing in eukaryotic chemotaxis. Bull Math Biol 65(1):95-128

19. Levchenko A, Iglesias PA (2002) Models of eukaryotic gradient sensing: application to chemotaxis of amoebae and neutrophils. Biophys J 82(1):50-63

20. Levine H, Aranson I, Tsimring L, Truong TV (1996) Positive genetic feedback governs cAMP spiral wave formation in Dictyostelium. Proc Nat Acad Sci 93(13):6382-6386

21. MacKay SA (1978) Computer simulation of aggregation in Dictyostelium discoideum. J Cell Sci 33(1):1-16

22. Martiel JL, Goldbeter A (1987) A model based on receptor desensitization for cyclic AMP signaling in Dictyostelium cells. Biophys J 52(5):807-828

23. Mathews N, Christensen AL, O'Grady R, Mondada F, Dorigo M (2017) Mergeable nervous systems for robots. Nat Commun $8(1): 439$

24. Noorbakhsh J, Schwab DJ, Sgro AE, Gregor T, Mehta P (2015) Modeling oscillations and spiral waves in Dictyostelium populations. Phys Rev E 91(6):62,711

25. Parent CA, Devreotes PN (1999) A cell's sense of direction. Science 284(5415):765-770

26. Parhizkar M (2020) Higher-order emergence in collective AI systems from computational model of Dictyostelium discoideum to swarm robotics. Ph.D. thesis, Geneva School of Social Sciences, Information systems, University of Geneva

27. Parhizkar M, Di Marzo Serugendo G (2015) Social amoeba Dictyostelium discoideum as an Inspiration for swarm robotics. In: IEEE 9th International Conference on self-adaptive and selforganizing systems (SASO), 2015, pp 162-163

28. Parhizkar M, Di Marzo Serugendo G (2017) An agent-based model for collective behaviors of social amoeba Dictyostelium 
discoideum morphogenesis: aggregation phase. In: International Conference on : SWARM'17

29. Parhizkar M, Di Marzo Serugendo G (2018) Agent-based models for first- and second-order emergent collective behaviours of social amoeba Dictyostelium discoideum aggregation and migration phases. Artif Life Robot Springer 23(4):498-507

30. Parhizkar M, Di Marzo Serugendo G, Hassas S (2019) Leaders and followers: a design pattern for second-order emergence. In: IEEE 4th Workshops on Foundations and Applications of Self* Systems (FAS* W). Umea

31. Parhizkar M, Nitschke J, Hellequin L, Di Marzo Serugendo G, Soldati T (2019) Self-organising agent-based model to study stream-breaking phenomenon during aggregation phase of Dictyostelium discoideum. In: International Conference on : SWARM'19

32. Rubenstein M, Ahler C, Nagpal R (2012) Kilobot: a low cost scalable robot system for collective behaviors. Robotics and Automation (ICRA), 2012 IEEE International Conference pp. 3293-3298

33. Rubenstein M, Cornejo A, Nagpal R (2014) Programmable selfassembly in a thousand-robot swarm. Science 345(6198):795-799

34. Schmickl T, Crailsheim K (2008) Trophallaxis within a robotic swarm: bio-inspired communication among robots in a swarm. Auton Robots 25(1):171-188

35. Seyfried J, Szymanski M, Bender N, Estaña R, Thiel M, Wörn H (2005) The I-SWARM project: intelligent small world autonomous robots for micro-manipulation. In: Lect. Notes Comput. Sci. https://doi.org/10.1007/978-3-540-30552-1_7

36. Umeda T, Inouye K (1999) Theoretical model for morphogenesis and cell sorting in Dictyostelium discoideum. Phys D 126(3-4):189-200

37. Valdastri P, Corradi P, Menciassi A, Schmickl T, Crailsheim K, Seyfried J, Dario P (2006) Micromanipulation, communication and swarm intelligence issues in a swarm microrobotic platform. Rob Auton Syst. https://doi.org/10.1016/j.robot.2006.05.001

38. Valentini G, Hamann H, Dorigo M (2015) Efficient decision-making in a self-organizing robot swarm: On the speed versus accuracy trade-off. In: Proc. 2015 Int. Conf. Auton. Agents Multiagent Syst., pp. 1305-1314. International Foundation for Autonomous Agents and Multiagent Systems

39. Valentini Gabriele HH, Dorigo M (2015) Efficient decision-making in a self-organizing robot swarm: On the speed versus accuracy trade-off. In: Proceedings of the 2015 International Conference on autonomous agents and multiagent systems. International Foundation for Autonomous Agents and Multiagent Systems, AAMAS, pp 1305-1314

40. Van Oss C, Panfilov A, Hogeweg P, Siegert F, Weijer CJ (1996) Spatial pattern formation during aggregation of the slime mould Dictyostelium discoideum. J Theor Biol 181(3):203-213

41. Vasieva OO, Vasiev BN, Karpov VA, Zaikin AN (1994) A model of Dictyostelium discoideum aggregation. J Theor Biol 171(4):361-367

42. Weijer CJ (2004) Dictyostelium morphogenesis. Curr Opin Genet Dev 14(4):392-398

43. Yu CH, Werfel J, Nagpal R (2010) Collective decision-making in multi-agent systems by implicit leadership. In: Proc. 9th Int. Conf. Auton. agents multiagent syst. Vol. 3-Volume 3, pp. 1189-1196. International Foundation for Autonomous Agents and Multiagent Systems

Publisher's Note Springer Nature remains neutral with regard to jurisdictional claims in published maps and institutional affiliations. 